\title{
Almost-common path interferometer using the separation of polarization states for digital phase-shifting shearography
}

\author{
Vanessa Rosso \\ Yvon Renotte \\ Serge Habraken, MEMBER SPIE \\ Yves Lion \\ Université de Liège \\ Department of Physics HOLOLAB \\ Bat. B5a, B-4000 Liège, Belgium \\ E-mail: vanessa.rosso@ulg.ac.be
}

Fabrice Michel, MEMBER SPIE

Vincent Moreau, MEMBER SPIE

Bernard Tilkens

DEIOS s.a.

Liège Science Park-Spatiopole

rue des Chasseurs Ardennais (WSL)

B-4031 Angleur, Belgium

\begin{abstract}
An original experimental setup for shearography with metrological applications is presented. The simplicity and the efficiency of the setup are provided by a shearing device, a prism that separates the TE and TM polarization modes with a coating and a thin glass plate placed on its face. The use of this shearing device enables an in-line and almost-common path configuration for the shearing interferometer, a path that leads to high stability and a low sensitivity to external disturbances. Moreover, the sensitivity of the interferometer can be easily adjusted for different applications by varying the shearing amount with glass plates of different thicknesses or by moving the shearing device between two lenses along the optical axis. The temporal phase-shifting method is applied through the use of a liquid crystal variable retarder. (c) 2007 Society of Photo-Optical Instrumentation Engineers. [DOI: 10.1117/1.2795632]
\end{abstract}

Subject terms: shearography; speckle interferometry; polarizing device; phase-shifting; liquid crystal retarder.

Paper 070099R received Feb. 6, 2007; revised manuscript received Apr. 19, 2007; accepted for publication Apr. 20, 2007; published online Oct. 19, 2007.

\section{Introduction}

Recently, optical techniques using temporal phase-shifting, involving techniques such as digital speckle pattern interferometry, ${ }^{1,2}$ digital shearography, ${ }^{3}$ and fringe projection, ${ }^{4}$ have emerged as new and efficient techniques with nondestructive metrological applications, e.g., 3-D shape measurement, surface displacement determination, and strain field and vibrational analysis. Shearography, or speckle shearing interferometry, is a full-field noncontact optical technique generally used for the measurement of the gradient of the displacement of a mechanically or thermally loaded surface. ${ }^{3,5-7}$ Shearography, as for any technique based on speckle interferometry, is used to have a better resolution on the measured strains ${ }^{8}\left(10^{-5}\right)$ than other technique such as the image correlation $\left(10^{-2}, 10^{-3}\right)$. Recent studies have also demonstrated the value of shearography for surface slope and shape measurements. ${ }^{9,10}$ Extensive work has been reported in this area and, further, different devices for the adaptation to shearography of the different types of shear encountered in conventional interferometry have been reported. ${ }^{11,12}$ Some cases are listed hereafter.

Shearing interferometers can be divided into two types: first, those with almost-common optical path interferometers, e.g., those with a glass edge or a Wollaston prism as a shearing device, ${ }^{6,9,13}$ and second, those that use a modified Michelson interferometer. ${ }^{14}$ In the first group, the two arms corresponding to the object beam and the reference beam are collinear and thus superimposed in one arm. Such a configuration enables the use of a laser with a low coherence length. This feature also makes the almost-common path interferometer less sensitive to external disturbances

0091-3286/2007/\$25.00 @ 2007 SPIE and, thus, more convenient for industrial uses. However, the sensitivity of typical almost-common path interferometers is limited by the shearing amount that may be imposed by the shearing device, e.g., a birefringent crystal ${ }^{6,7}$ and, as a consequence, it may be suitable for only a few applications. This problem doesn't appear with the Michelson-type interferometer because the shearing amount can be easily modified. ${ }^{14}$

In this paper, an original setup for digital phase-shifting shearography is presented. The key element of the experimental setup is the shearing device, a prism with a coating and a thin glass attached plate that separates the two TE and TM polarization modes. This shearing device yields an almost-common path and an in-line configuration of the shearing interferometer, an arrangement that leads to a compact device that is less sensitive to external disturbances. The separation of the polarization states has been chosen because it is a convenient method that enables one to always distinguish each interfering beam when they are collinear simply by the rotation of a polarizer. In the same way, the intensity of each interfering beam can be easily adjusted to have a high modulation in the resulting interferograms. Moreover, the sensitivity of the interferometer can be easily modified for different applications by varying the shearing amount with glass plates of different thicknesses or simply by moving the shearing device along the optical axis, as proposed by Valera and Jones. ${ }^{15}$ The phaseshifting method is accomplished by using a liquid crystal variable retarder, which enables an easy positioning and a good handiness in the experimental setup relative to other phase shifters such as a piezoelectric translator ${ }^{2,3}$ or a technique using a highly birefringent optical fiber. ${ }^{15,16}$

Finally, to demonstrate the efficiency of this shearing interferometer, an experiment consisting of centrally load- 
ing a steel plate was performed. The resulting out-of-plane displacement derivative relative to the shearing direction was determined.

\section{Digital Phase-Shifting Shearography}

Shearography is a useful tool for measuring the slope contours or the displacement derivatives of an object under a

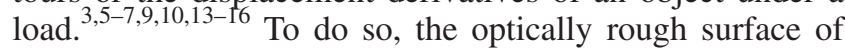
the object to be studied is irradiated with coherent light to create a speckle pattern. A digital camera (CCD or CMOS) records the interference between this object speckle pattern and an identical speckle pattern spatially displaced, or sheared, thanks to a shearing device; the resulting interferogram is called a shearogram. By comparing the shearograms recorded before and after loading of the object, fringes sensitive to the displacement derivative relative to the shearing direction can be obtained. Phase-shifting 2,3,17 and unwrapping ${ }^{18-20}$ techniques are usually used to process the phase information from these fringes to determine quantitatively the desired metrological parameter. In this paper, the general four-buckets ${ }^{17}$ algorithm and the growing region ${ }^{20}$ method were used, respectively, for phase-shifting and phase unwrapping.

In shearography, the unwrapped phase map $\Delta_{\text {unwrapped }}(x, y)$ obtained thanks to the temporal phaseshifting technique is related to the first derivative of the displacement field relative to the shearing direction $X_{i}$ of the object speckle wavefront and described in good approximation, when the shearing amount $\delta x_{i}$ is small, by the following relationship: ${ }^{3}$

$\Delta_{\text {unwrapped }}=\left[\left(\mathbf{K e}_{\mathbf{X}}\right) \frac{\partial u}{\partial x_{i}}+\left(\mathbf{K e}_{\mathbf{Y}}\right) \frac{\partial v}{\partial x_{i}}+\left(\mathbf{K e}_{\mathbf{Z}}\right) \frac{\partial w}{\partial x_{i}}\right] \delta x_{i}$,

where $\mathbf{K}$ is the sensitivity vector of the interferometer and $\mathbf{e}_{\mathbf{X}}, \mathbf{e}_{\mathbf{Y}}$, and $\mathbf{e}_{\mathbf{Z}}$ are the unit vectors, respectively, along the $X, Y$, and $Z$ directions. In the orthogonal coordinate system $X Y Z$, the $X$ direction is horizontal and the $Y$ direction is vertical. The $Z$ direction is from the object to the camera, it is out-of-plane and normal to the surface of the object (see Fig. 5 in Sec. 3.3). Also, $u, v$, and $w$ are the components of the displacement vector $\mathbf{u}$, respectively, along the $X, Y$, and $Z$ directions. In this paper, the efficiency of the experimental approach is demonstrated for the case of a pure out-ofplane displacement derivative measurement, i.e., the sensitivity vector $\mathbf{K}$ is orthogonal to the object plane $(X, Y)$. The resulting posttreatment of the phase distribution, $\Delta_{\text {unwrapped }}(x, y)$, is then summarized by

$\Delta_{\text {unwrapped }}=\left(\mathbf{K e}_{\mathbf{Z}}\right) \frac{\partial w}{\partial x_{i}} \delta x_{i}$

The out-of-plane displacement derivative relative to the shearing direction can be determined from Eq. (2).

\section{Experimental Details}

\subsection{Shearing Device}

The shearing device used in digital shearography splits the light coming from one object point to give two image points in the image plane. A pair of laterally sheared images is thus received by the image sensor. In other words, the shearing device brings light scattered from two neighboring points $P(x, y, z)$ and $P(x+\delta x, y, z)$ on the object surface to interfere at a point on the image plane of a camera. In this case, the shearing is assumed to be parallel to the $X$ axis, and $\delta x$ is the shearing amount.

Different shearing devices based on the separation of polarization states are presented in the literature. However, with those shearing devices, either the shearing amount is fixed, e.g., with a double-refractive prism such a Wollaston prism, ${ }^{6,9}$ or the related phase-shifting technique is not easily implemented or requires a careful control, e.g., phase steps produced by straining a highly birefringent fiber ${ }^{15}$ or by tuning the laser emission wavelength. ${ }^{16}$

The key element of this shearing experiment is the shearing prism that separates the two polarization modes. The prism separates an incident wave into two orthogonal linearly polarized waves, a transverse electric (TE) or $s$-wave and a transverse magnetic (TM) or $p$-wave. The separation of the two polarization states is produced by a coating on the back face of a glass prism obtained from Edmund Optics. Theoretically the separation of the polarized states is optimal for a normal incidence on the front face of the prism. In this case, the emergent polarized TE and TM waves correspond, respectively, to the beams reflected and transmitted by the coating, see Fig. 1(a). The behavior of the prism was checked by measuring the reflectance and transmittance of the TE and TM polarized incident waves (see Fig. 2). This figure indicates that at normal incidence, the separation of the two polarization modes can reach 93\%. However, the separation is optimal for incidence angles ranging from 6 to $22 \mathrm{deg}$. To work in this optimal range, an incidence angle of 10 above 0 deg has been used herein [see Fig. 1(a)].

To direct the TE and TM emergent waves in the same direction, a thin glass plate is attached on the coating of the prism with an oil of the same refractive index as the prism and the glass plate, $n=1.52$, ensuring the continuity of the media. This glass plate directs by total reflection the TM polarized wave transmitted by the coating in the same direction as the reflected TE polarized wave. In this way, if the shearing is assumed to be parallel to the $X$ axis, the TE and TM waves are separated by $\delta x$, a distance that is proportional to the thickness of the glass plate [see Fig. 1(b)]. To produce interference between the two beams, a linear polarizer oriented between the TE and TM polarization directions must be introduced into the optical path of the parallel TE and TM beams. In practice, the orientation of the polarizer is chosen to equalize the intensities of the two interfering beams to have a high modulation in the resulting interferograms.

If the refraction through the coating and the thickness induced by the oil are neglected, we can follow the optical path of a ray of light with an incidence angle $\theta_{i}$ on the front face of the prism [see Fig. 1(c)] in using the laws of refraction $\left(\theta_{r}\right.$ is the refraction angle) and reflection. If the refractive index of the prism follows the condition $n \geqslant \sqrt{2}$, the use of these laws enable the determination of the relationship between the shearing amount $\delta x$, the thickness of the glass plate $d$, and the incidence angle $\theta_{i}$ [see Figs. 1(c) and Fig. 3]: 

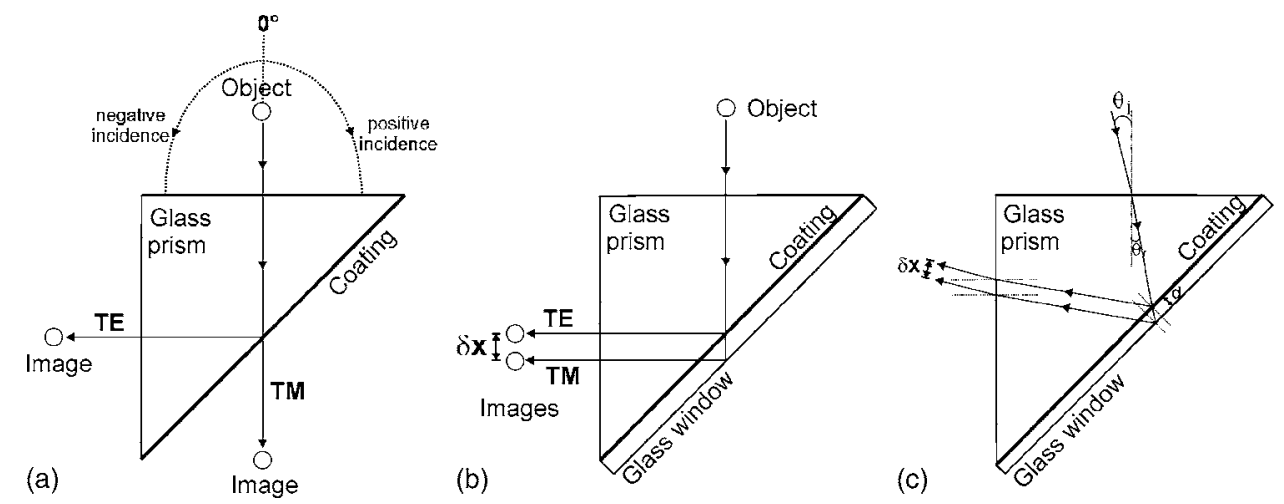

Fig. 1 (a) Schematic of the separation of an incident light beam into two TE and TM polarized beams as a result of a coating on the back face of a prism and (b) schematic of the shearing prism. To direct the TE and TM emergent waves in the same direction, a thin glass plate is attached to the coating of the prism with an oil whose refractive index $n$ is 1.52. The TE and TM polarized beams are sheared by $\delta x$, a distance that is proportional to the thickness of the glass. (c) Schematic of the optical path followed through the shearing prism by a ray of light with an incidence angle $\theta_{i}$ on the front face and giving rise to a shearing amount depending on the incidence angle $\theta_{i}$ and the thickness $d$ of the glass plate.

$\delta x=\sqrt{2} d \cos \theta_{i}\left(1+\frac{n \sin \theta_{i}}{n^{2}-\sin ^{2} \theta_{i}}\right)$.

At normal incidence on the front face of the prism, $\theta_{i}=0$. Equation (3) is simplified and the shearing amount is $\delta x$ $=\sqrt{2} d$.

\subsection{Phase Modulator: A Liquid Crystal Variable Retarder}

As with any interferometric technique that uses the temporal phase-shifting method, it is necessary to record some interferograms that are phase shifted for comparison with others. To produce this phase shift, it is necessary to introduce a phase modulator into the experimental setup. In most of those experiments, a piezoelectric translator ${ }^{2,3}$ (PZT) is used for phase modulation. In contrast, for the interferometric experiments based on the separation of the TE and TM polarization states, the most reported phase

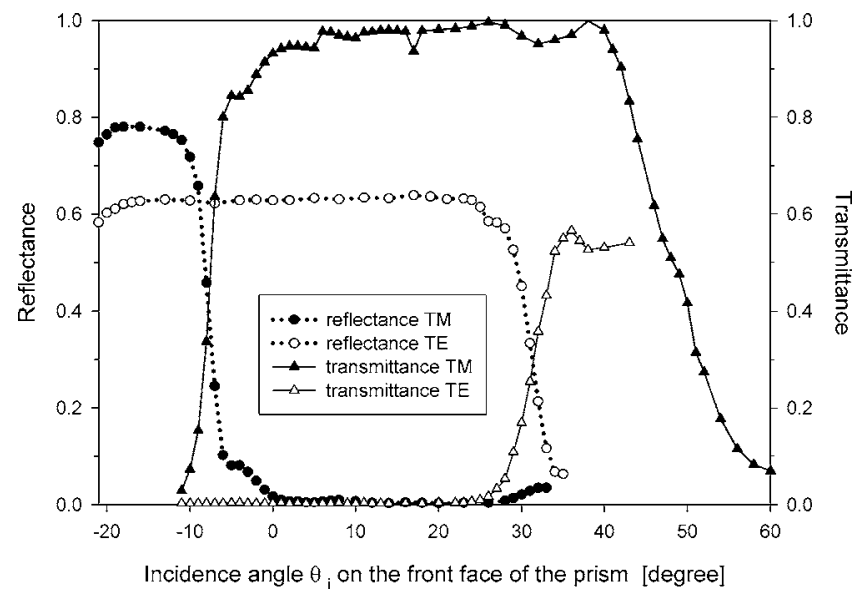

Fig. 2 Transmittance and reflectance of the TE and TM waves obtained at $\lambda=532 \mathrm{~nm}$ as a function of the angle of incidence on the front face of the shearing prism. The sign of the angle of incidence is defined in Fig. 1(a). modulators $^{2}$ are liquid crystal retarders ${ }^{21}$ or are based on highly birefringent optical fibers. ${ }^{15,16}$ Contrary to the fiberbased phase modulator, the liquid crystal variable retarder presents the advantages of being very compact and so can be introduced in transmission anywhere in the experimental setup. Moreover, it is less temperature dependent.

In our experimental shearography setup, which is described in Sec. 3.3, a liquid crystal variable retarder from Meadowlark Optics (model LVR-200 with basic controller D3040, phase uncertainty: ${ }^{21} \lambda / 28$ ) is used to perform the phase-shifting technique. When combined with other optical components, liquid crystal variable retarders produce electrically controllable attenuation, linear polarization rotation, or phase modulation. Pure phase modulation is accomplished by aligning the optical axis of the liquid crystal

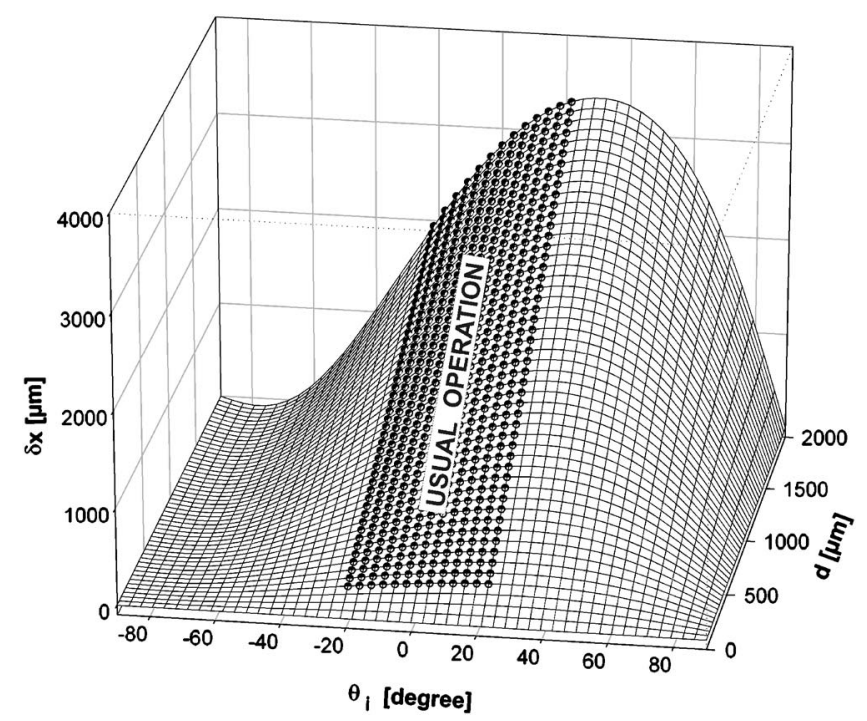

Fig. 3 Evolution of the shearing amount, $\delta x$ as a function of both the thickness $d$ of the glass plate placed on the coating of the prism and the incidence angle $\theta_{i}$. The blackened area represents the usual sensor operation parameters. 


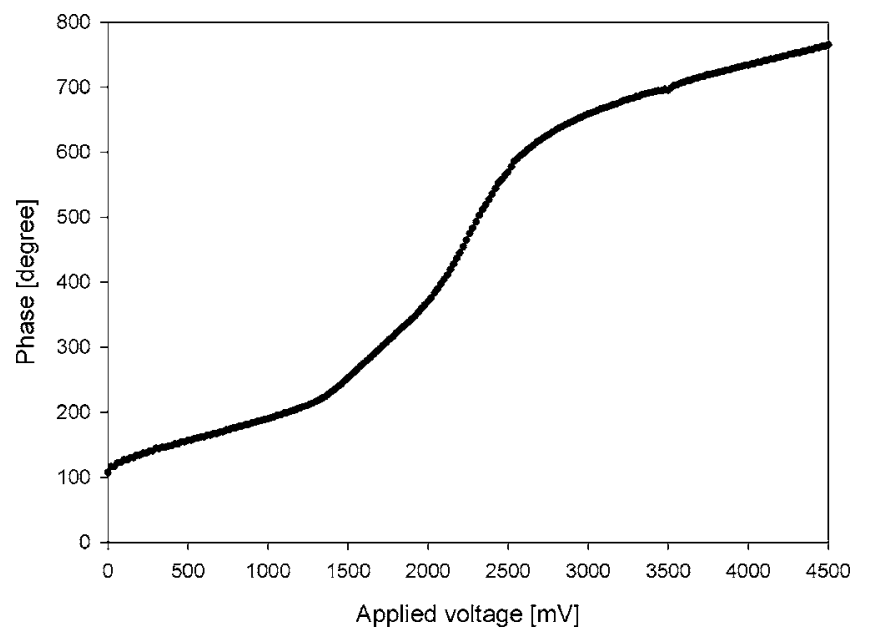

Fig. 4 Calibration curve of the phase modulator, a liquid crystal variable retarder, obtained with the experimental shearography setup. In the case of the four-buckets algorithm, the incremental phase $\alpha$ $=\pi / 2$ is obtained by applying, for example, successively 1500 , 1890,2166 , and $2371 \mathrm{mV}$ to the liquid crystal cell.

retarder parallel to a linearly polarized input beam. Such a phase modulator uses nematic liquid crystal materials to electrically control polarization. It provides tunable retardation by changing the effective birefringence of the material with an applied voltage.

The calibration of the phase modulator is accomplished by determining the phase shift it induces versus the applied voltage. The liquid crystal variable retarder is well known for exhibiting a high nonlinearity versus the applied voltage, and thus, it has been calibrated in situ, following the approach of Ochoa and Huntley. ${ }^{22}$ This consists of recording two images, namely, one before and one after applying a known voltage step to the phase modulator. The mean square intensity difference between the two images, obtained by averaging over all of the pixels in the image, can then be plotted as a function of $V$, the voltage applied while recording the second image. Figure 4 shows the resulting calibration curve of the liquid crystal variable retarder obtained with the experimental setup of shearography that is described in Sec. 3.3.

\subsection{Almost-Common Path Interferometer for Shearography}

The design of the experimental setup, which uses a pure out-of-plane digital shearography arrangement is illustrated in Fig. 5. The $532 \mathrm{~nm}$ wavelength light comes from a NdYAG laser with a total output power of $30 \mathrm{~mW}$. The object speckle pattern was produced by retroscattering of the incident light from a test object. The shearograms, recorded by a CMOS camera (from Lumenera Corporation, model LU105C: 15 frames per second at full $1280 \times 1024$ resolution), arise from the interference between the object speckle wavefront and the same speckle wavefront shifted spatially along the $X$ axis by the shearing device. The behavior of the latter is explained in Sec. 3.1. A second prism was attached with oil with a refractive index $n=1.52$ on the shearing device (Fig. 5) to make the interferometer compact and in-line. The second prism is a traditional nonpolarizing

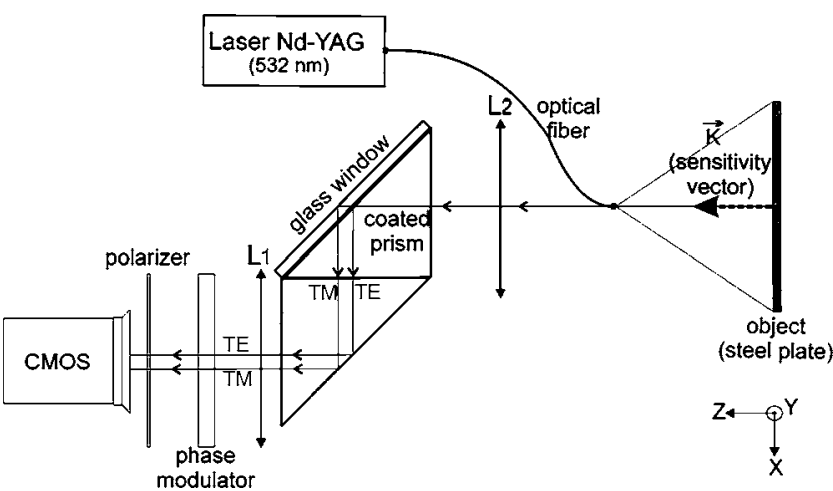

Fig. 5 Experimental setup of the out-of-plane almost-common path interferometer used for shearography.

glass prism but is silver coated for better reflectivity; it has the same dimensions as the first shearing prism. To produce interference between the two emergent polarized beams, a linear polarizer oriented between the TE and TM polarization directions is introduced in front of the objective of the CMOS camera. A set of four shearograms, phase shifted with respect to one another, are recorded for a state of the studied object by varying the voltage applied to the liquid crystal variable retarder that was placed between the second prism and the polarizer. The slow and fast axes of the phase modulator are oriented, respectively, along the TE and TM polarization directions.

Finally, two identical lenses $L_{1}$ and $L_{2}$ with an effective focal length of $6 \mathrm{~cm}$ were introduced into the experimental setup (see Fig. 5). The role of $L_{1}$ is to focus the TM speckle wavefront on the glass-air interface of the shearing device and, consequently, to avoid any variation in the shearing amount as the result of a nonuniform thickness of the thin glass plate. The second lens, $L_{2}$, which defocuses the waves, also increases the shearing amount in proportion to its magnification $M_{\text {lens }}$, and thus, the shearing amount behind $L_{2}$ is

$\delta x_{\text {lens }}=M_{\text {lens }} \delta x$.

To simplify the following notation, the value $\delta x_{\text {lens }}$ of the shearing amount behind lens $L_{2}$ will simply be referred to as $\delta x$.

The shearing amount can be changed by replacing the thin glass plate attached to the coating of the prism by another with a different thickness $d$. On the other hand, according to Eq. (4), the shearing amount varies with the magnification $M_{\text {lens }}$ that can be modified by moving the shearing device between the two lenses $L_{1}$ and $L_{2}$, as proposed by Valera and Jones. 15

The resulting experimental setup is an almost-common path interferometer, i.e., the object and reference beams follow almost the same geometric path before interference. Thus, because the two interfering beams would be equally disturbed, this interferometer has the advantage of being less sensitive to external disturbances than traditional digital speckle interferometers. Moreover, this shearographic interferometer is compact and uses a CMOS camera, knowing that CMOS technology is well recognized today to be implemented in low-cost commercial devices. It also uses a 


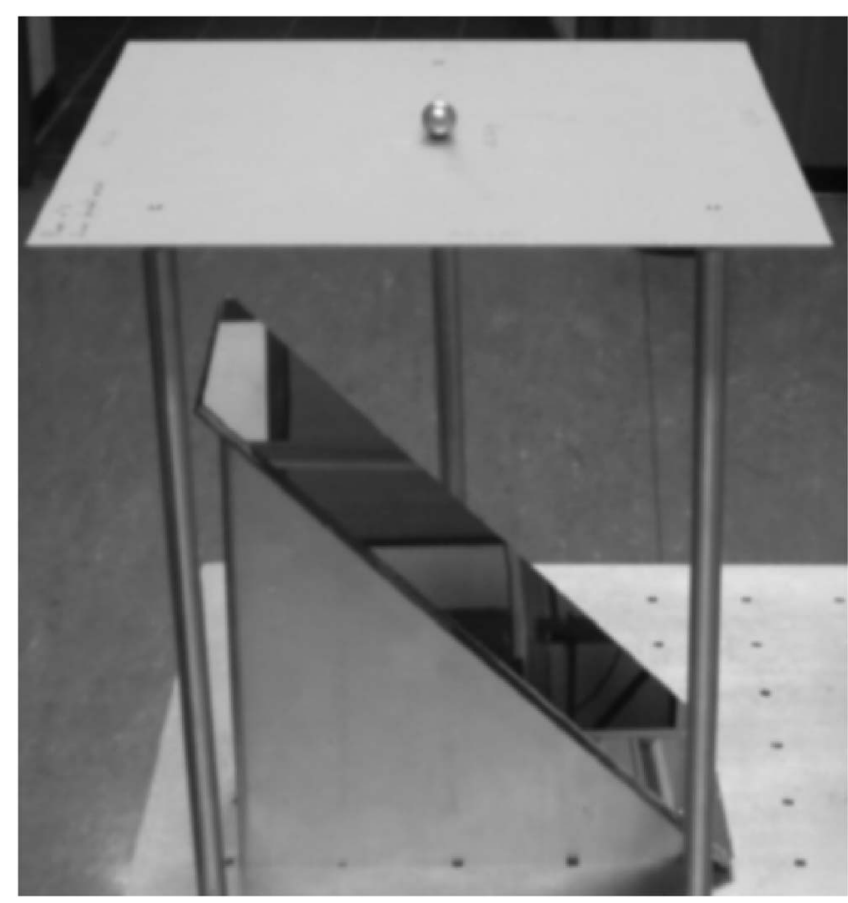

Fig. 6 Photograph illustrating the central loading of a steel plate with a $12 \mathrm{~g}$ ball.

liquid crystal variable retarder that does not present a hysteresis effect, while a PZT must be compensated and thus makes it more expensive to eliminate the hysteresis effect, ${ }^{21}$ and that is almost 15 times faster than a PZT with almost the same resolution ${ }^{21}$ (phase uncertainty $\approx \lambda / 25$ ).

\subsection{Studied Object and the Loading System}

The object to be studied is a $300 \times 300 \times 1 \mathrm{~mm}^{3}$ steel plate pierced by three holes in an isosceles triangle arrangement, holes that will become the three points supporting the plate. The steel plate was placed on three balls held by three steel posts screwed into an optical table, as shown in Fig. 6. A fourth smaller hole is placed at the center of gravity of the isosceles triangle to hold, in a reproducible fashion, a ball of known mass $m$. The plate is thus centrally loaded and the applied force $F$ is equal to the weight of the ball, i.e., $0.118 \mathrm{~N}$ for a ball with a mass of $12 \mathrm{~g}$. This horizontal object-loading system can be used thanks to a mirror at 45 deg placed under the steel plate (see Fig. 6) that reorients the light along the out-of-plane $Z$ direction. However, to not complicate Fig. 5, a usual vertical object-loading system is represented in the $(X, Z)$ plane.

Only a portion of the steel plate was scanned by the shearographic interferometer so, in the following, the studied area of the steel plate is a square with a side of $87 \mathrm{~mm}$ centered on the load.

\section{Results and Discussion}

The wrapped phase map (see Fig. 7) was obtained with the four-buckets algorithm ${ }^{17}$ applied to the phase-shifted shearograms recorded for the steel plate before and after loading. The wrapped phase map of Fig. 7 was obtained with the experimental setup shown in Fig. 5 for a shearing of $8.4 \mathrm{~mm}$, corresponding to a 0.35 -mm-thick glass plate

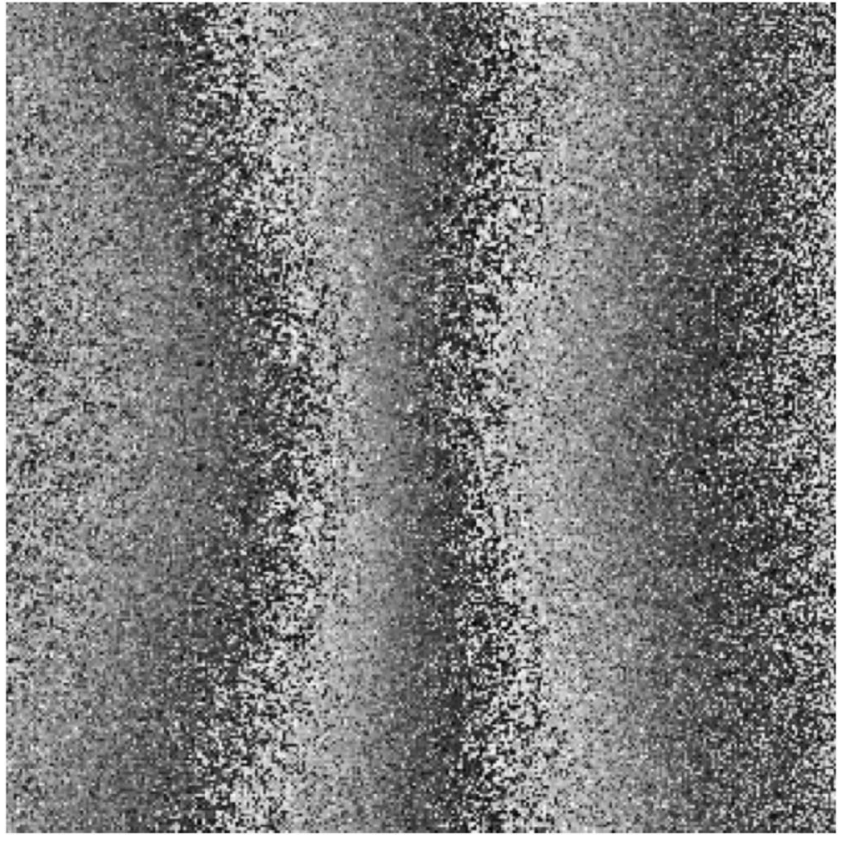

Fig. 7 Wrapped phase map obtained with the shearographic interferometer illustrated in Fig. 5 and the loading illustrated in Fig. 6. The shearing amount $\delta x$ is $8.4 \mathrm{~mm}$ and the thickness of the glass plate is $d=0.35 \mathrm{~mm}$.

and $M_{\text {lens }}=17$, and the $12 \mathrm{~g}$ loading shown in Fig. 6. Before unwrapping the phase map, the noise was reduced by filtering with a convolution mask of dimension three applied at the sine/cosine ${ }^{18,23,24}$ level. The filtered wrapped phase map is shown in Fig. 8 and the corresponding phase unwrapped by the region-growing technique in Fig. 9. According to Eq. (2), the out-of-plane displacement derivative

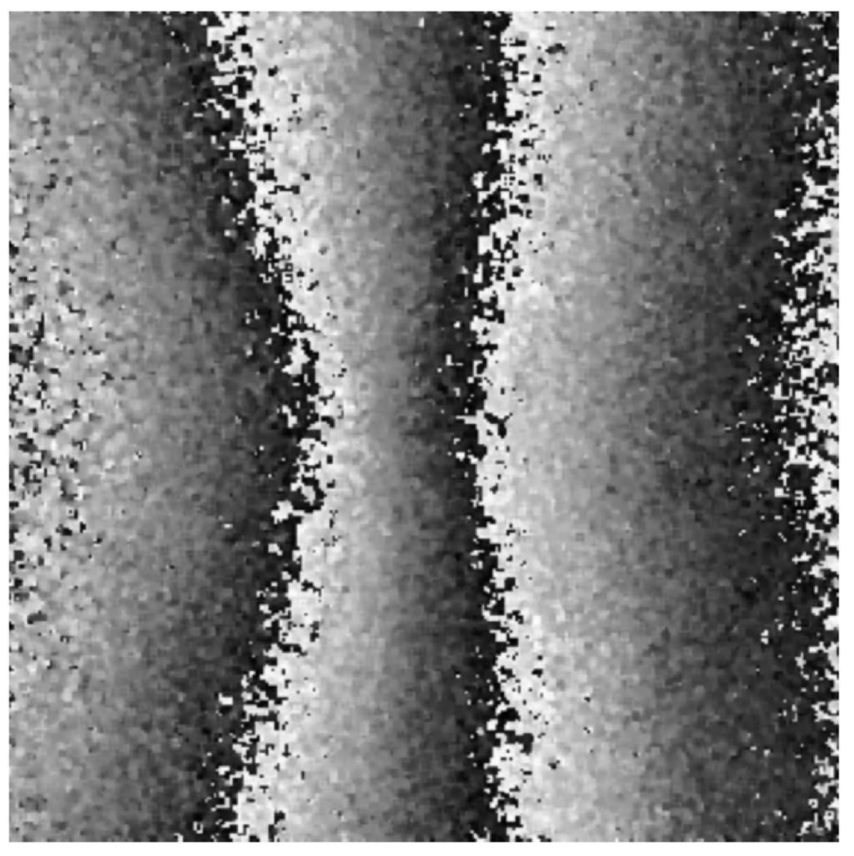

Fig. 8 Wrapped phase map of Fig. 7 filtered once at the sine/cosine level by a mean mask of dimension three. 


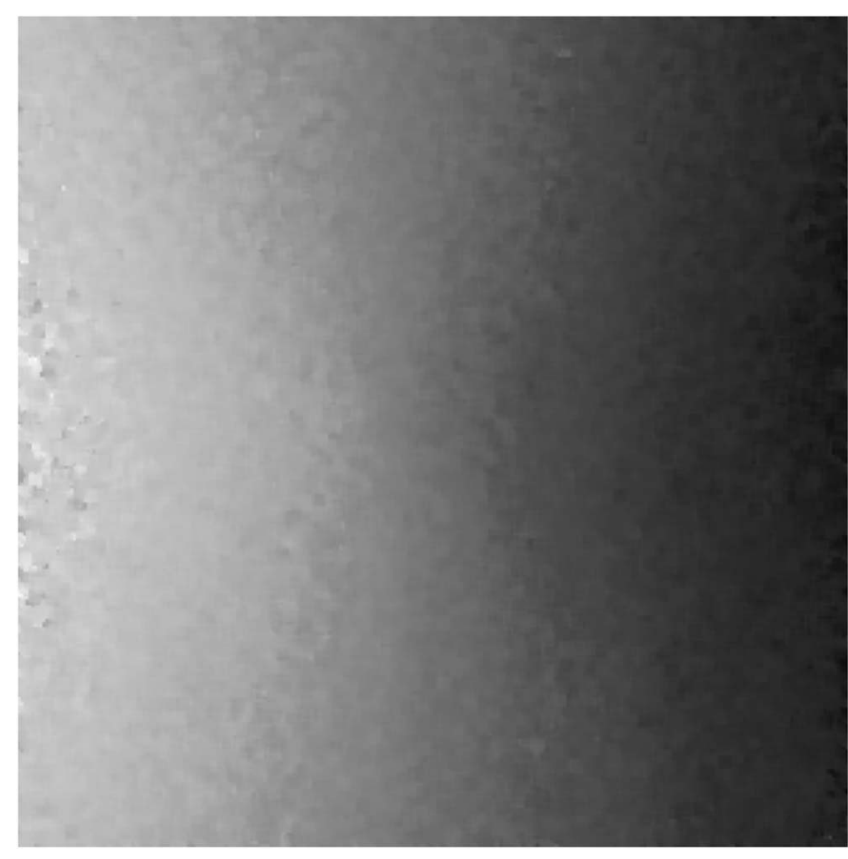

Fig. 9 Phase map of Fig. 8 unwrapped by the region-growing technique.

relative to the $X$ direction, $\partial w / \partial x$, can be determined from the filtered and unwrapped phase map, Fig. 9; the quantitative results are presented in three dimensions in Fig. 10. A detailed error analysis yields to a relative uncertainty of $8 \%$ on the results shown in Fig. 10, due to the phase uncertainty and mainly to the relative uncertainty on the shearing amount (up to $8 \%$ ).

If a heavier load is used, the number of fringes observed in the wrapped phase map naturally increases (see Fig. 11) until the contrast of the fringes suddenly decreases because of the high spatial frequency of the fringes and the noise present in the image. Contrary to most almost-common

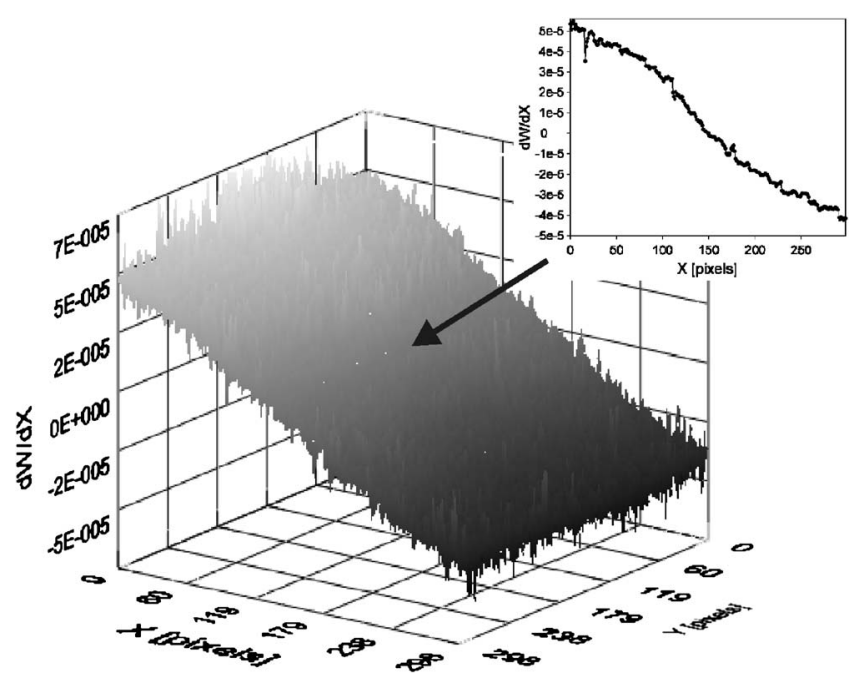

Fig. 10 Out-of-plane displacement derivative relative to the $X$ direction, $\partial w / \partial x$, determined from the unwrapped phase map of Fig. 9 and the corresponding profile along the shearing $X$ direction, passing by the loading point.

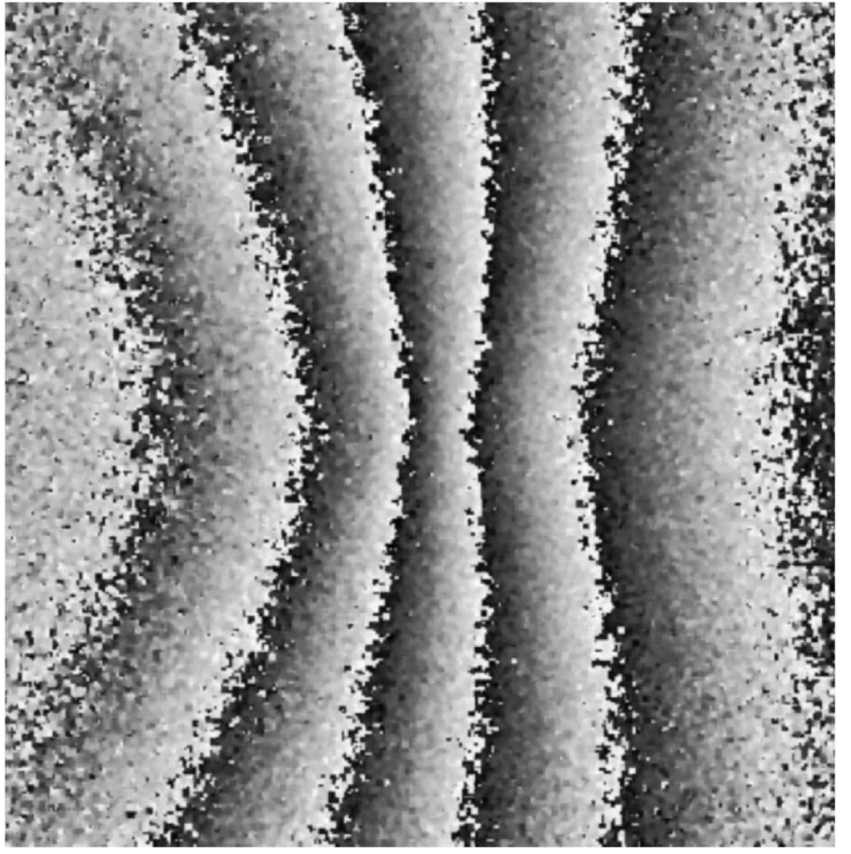

Fig. 11 Filtered (mean mask of dimension 3) and wrapped phase map obtained with the shearographic interferometer of Fig. 5 with $\delta x=8.4 \mathrm{~mm}$ and the loading system of Fig. 6 , but with a ball with a mass of $23 \mathrm{~g}$.

path interferometers in which the shearing amount is fixed, in this interferometer, the sensitivity can be modified by varying the shearing amount. Figure 12 shows the wrapped phase maps obtained with the 12-g loading shown in Fig. 6 and the experimental design of Fig. 5, but with a thinner, $0.16 \mathrm{~mm}$, and a thicker, $0.56 \mathrm{~mm}$, glass plate, yielding shearings of 3.9 and $13.6 \mathrm{~mm}$, respectively $\left(M_{\text {lens }}=17\right)$. As predicted by Eq. (2), a suitable number of fringes to maintain a good contrast can be obtained by using a short shearing amount, if the strain is important, and a longer amount, if the load is small.

Consequently, this interferometer maintains both the advantages of the Michelson-type interferometer because it enables one to adjust its sensitivity for different applica-

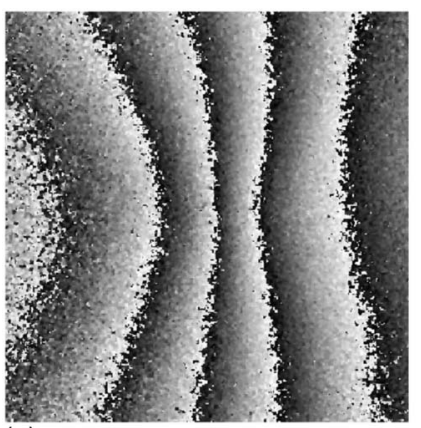

(a)

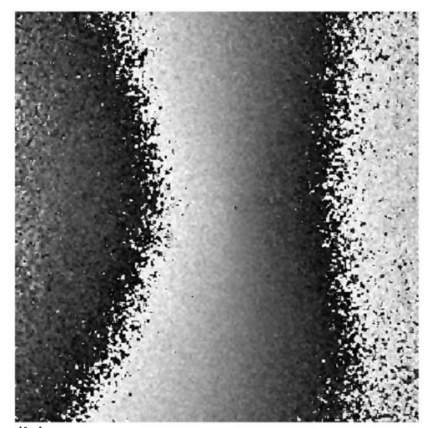

(b)
Fig. 12 Filtered (mean mask of dimension 3) and wrapped phase map obtained with the loading system of Fig. 6 and a 12-g ball and the shearographic interferometer of Fig. 5, but with different shearing amounts obtained with glass plates of different thicknesses: (a) $\delta x=13.6 \mathrm{~mm}(d=0.56 \mathrm{~mm})$ and (b) $\delta x=3.9 \mathrm{~mm}(d=0.16 \mathrm{~mm})$. 
tions by varying the shearing amount, and the advantage of an almost-common path interferometer, a compact in-line experimental setup with good stability to external disturbances.

\section{Conclusions}

An almost-common path shearographic interferometer for applications in temporal phase-shifting interferometry was developed and the behavior of the main elements constituting the interferometer were studied. The shearing device, which is the key element of the interferometer, is a prism that separates the TE and TM polarization states with a coating and an attached thin glass plate.

This shearing device and the use of components such as a CMOS camera and a liquid crystal variable retarder enable a compact, in-line, and low-cost almost-common path experimental setup that is less sensitive to external disturbances and that enables one to adjust its sensitivity for different applications by varying the shearing amount.

Finally, phase maps were presented that prove the efficiency of the experimental setup for the case of an out-ofplane displacement derivative measurement.

\section{Acknowledgments}

Fabrice Michel and Vincent Moreau were supported by a "FIRST" project grant by the Region Wallonne Government (DGTRE). The authors thank both the Centre Européen d'Archéométrie-University of Liège-and the Communauté Française for their support.

\section{References}

1. R. Jones and C. Wykes, Holographic and Speckle Interferometry Cambridge Univ. Press, Cambridge (1989).

2. P. K. Rastogi, Digital Speckle Pattern Interferometry and Related Techniques, John Wiley \& Sons, Chichester (2001).

3. W. Steinchen and L. Yang, Digital Shearography, SPIE Press, Bellingham, WA (2003).

4. C. A. Walker, Ed., Handbook of Moiré Measurement, Series in Optics and Optoelectronics, IOP Publishing Ltd., Bristol (2004).

5. Y. Y. Hung, "A speckle-shearing interferometer: A tool for measuring derivatives of surface displacements," Opt. Commun. 11(2), 132-135 (1974).

6. V. M. Murukeshan, O. L. Seng, and A. Asundi, "Polarization phase shifting shearography for optical metrological applications," Opt. Laser Technol. 30(8), 527-531 (1998).

7. Y. Y. Hung, "Applications of digital shearography for testing of composite structures," Composites, Part B 30(7), 765-773 (1999).

8. G. Montay, M. François, M. Tourneix, B. Guelorget, C. VialEdwards, and I. Lira, "Analysis of plastic strain localization by a combination of the speckle interferometry with the bulge test," Opt. Lasers Eng. 45(1), 222-228 (2007).

9. H. M. Shang, Y. Y. Hung, W. D. Luo, and F. Chen, "Surface profiling using shearography," Opt. Eng. 39(1), 23-31 (2000).

10. R. M. Groves, S. W. James, and R. P. Tatam, "Shape and slope measurement by source displacement in shearography," Opt. Lasers Eng. 41(4), 621-634 (2004).

11. J. C. Wyant, "Double frequency grating lateral shear interferometer," Appl. Opt. 12(9), 2057-2060 (1973).

12. K. Matsuda and M. Namiki, "Holographic lateral shear interferometer for differential interference contrast method," J. Opt. 11, 81-85 (1980).

13. Y. Y. Hung and C. E. Taylor, "Measurement of slopes of structural deflections by speckle-shearing interferometry," Exp. Mech. 14(7), 281-285 (1974).

14. A. R. Ganesan, D. K. Sharma, and M. P. Kothiyal, "Universal digital speckle shearing interferometer," Appl. Opt. 27(22), 4731-4734 (1988).

15. J. D. Valera and J. D. C. Jones, "Phase stepping in fiber-based speckle shearing interferometry," Opt. Lett. 19(15), 1161-1163 (1994).

16. R. M. Groves, S. W. James, and R. P. Tatam, "Polarizationmultiplexed and phase-stepped fibre optic shearography using laser wavelength modulation," Meas. Sci. Technol. 11, 1389-1395 (2000).

17. K. Creath, "Phase-shifting speckle interferometry," Appl. Opt.
24(18), 3053-3058 (1985).

18. D. C. Ghiglia and M. D. Pritt, Two-Dimensional Phase Unwrapping: Theory, Algorithms, and Software, Wiley, New York (1998).

19. T. J. Flynn, "Two-dimensional phase unwrapping with minimum weighted discontinuity," J. Opt. Soc. Am. A 14(10), 2692-2701 (1997).

20. A. Baldi, "Phase unwrapping by region growing," Appl. Opt. 42(14), 2498-2505 (2003)

21. P. Slangen and B. Gautier, "Nematic liquid crystals light valve calibration and application to phase shifting speckle interferometry," in Speckle06: Speckles, From Grains to Flowers, P. Slangen and C. Cerruti, Eds., Proc. SPIE 6341, 63410C (2006).

22. N. A. Ochoa and J. M. Huntley, "Convenient method for calibrating nonlinear phase modulators for use in phase-shifting interferometry," Opt. Eng. 37(9), 2501-2505 (1998).

23. V. Rosso, F. Michel, V. Moreau, Y. Renotte, B. Tilkens, and Y. Lion, "Highlighting properties of filters for their application in temporal phase shifting interferometry," in Photonic Applications in Biosensing and Imaging, W. C. Chan, K. Yu, U. J. Krull, R. I. Hornsey, B. C. Wilson, and R. A. Weersink, Eds., Proc. SPIE 5969, 626-635 (2005).

24. H. A. Aebischer and S. Waldner, "A simple and effective method for filtering speckle-interferometric phase fringe patterns," Opt. Commun. 162(4-6), 205-210 (1999).

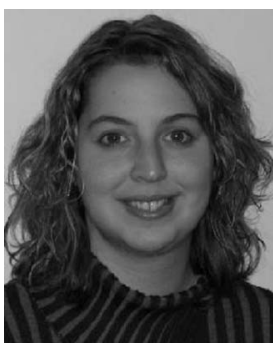

Vanessa Rosso received her BSc and MSc in physics from the University of Liège, Belgium, in 2002 and 2004, respectively. She joined the Laboratory of Holography (HOLOLAB) in 2001 where she has studied nonlinear optical effects in nanoparticles doped xerogels. Currently she is working on her PhD thesis about metrological applications of speckle interferometry and particularly shearography.

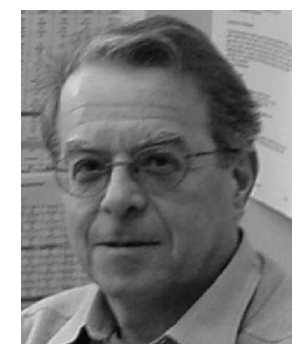

Yvon L. Renotte received his PhD in physics in 1973 from the State University of Liège, Belgium, where he taught optics to graduate and postgraduate students in the faculties of science and civil engineering. For many years, he worked on photographic processes in cooperation with Agfa-Gevaert Co., Antwerp, Belgium. He is currently involved in programs of holographic recording materials such as AgHal, dichromatedgelatine, photopolymers, and photoresists, optical metrology, and the study, fabrication, and testing of computer calculated holographic optical elements and guided-mode resonance filters. He is also a consultant for DEIOS, s.a., a spin-off developing 3D recording systems for archaeological and medical applications. He is a member of SPIE and ECO and is president of the Belgian Commission for Optics.

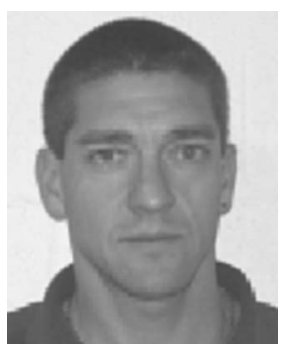

Serge Habraken received his $\mathrm{PhD}$ in physics in 1995 from the University of Liege, Belgium (ULg), and he researched diffractive optics and holography. After completing his thesis, he joined the Space Center of Liege (CSL) where he worked as project manager, then lead of a research and development group involved in microfabrication for optics. In 2005, he became a professor in the Physics Department of the ULg, sharing the direction of the HOLOLAB with Prof. Yves Lion. $\mathrm{He}$ is still attached to the CSL and manages the cross activities between both institutes. 


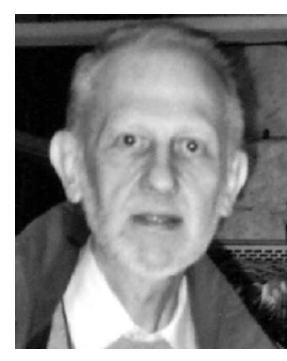

Yves Lion received a BSc and a PhD in physics from University of Liege, Belgium, in 1967 and 1971, respectively. He spent one year at the National Institutes of Health to study photochemical reactions by the technique of spin-trapping and electron spin resonance. $\mathrm{He}$ is a full professor and teaches physics with the faculty of civil engineering. $\mathrm{He}$ is also head of the HOLOLAB at the Physics Institute of Liege University.

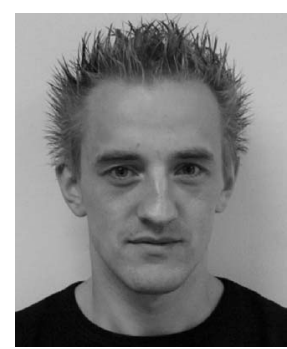

Fabrice Michel received his BSc in physics from the University of Liège in June 2004. His essay was related to diffractive optics and surface plasmon resonance. During the academic year 2004-2005, he supervised students from the Applied Sciences Faculty during their physics laboratories and he has begun to work on interferometry (shearography). Since then, he has been simultaneously working on his $\mathrm{PhD}$ thesis and in the research and development department of DEIOS, s.a. His research interests include non-destructive testing by shearography, thin-film optical systems, and optical engineering for industry.

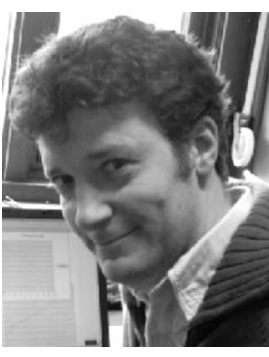

Vincent Moreau received his BEng, MSc, and $\mathrm{PhD}$ degrees in physics from the University of Liège, Belgium, in 1995, 1996 and 2001 respectively. $\mathrm{He}$ is currently the research and development manager of DEIOS, s.a. He also works for the Walloon Department of Research on diffractive optics applications in communication, metrology, and medicine.

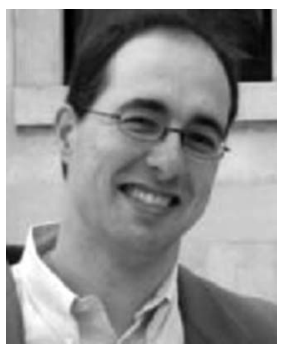

Bernard G. Tilkens received his BSc in physics in 1994, his MSc degree in optoelectronics in 1995, and his PhD in physics in 2003 from the University of Liège, Belgium. He also performed research in the HOLOLAB, Physics Institute of the University of Liège. Since 2004, he has been the CEO of DEIOS, s.a., a company involved in optical metrology and the engineering of dedicated optical solutions. 